\title{
BMJ Open Capturing patient-reported and quality of life outcomes with use of shorter regimens for drug-resistant tuberculosis: mixed-methods substudy protocol, TB PRACTECAL-PRO
}

\author{
Beverley Stringer (D) , ${ }^{1}$ Karen Lowton, ${ }^{2}$ Nicola James (i) , ${ }^{1}$ \\ Bern-Thomas Nyang'wa (D) 1,3
}

To cite: Stringer B, Lowton K, James $\mathrm{N}$, et al. Capturing patient-reported and quality of life outcomes with use of shorter regimens for drugresistant tuberculosis: mixedmethods substudy protocol, TB PRACTECAL-PRO. BMJ Open 2021;11:e043954. doi:10.1136/ bmjopen-2020-043954

- Prepublication history for this paper is available online. To view these files, please visit the journal online (http://dx.doi. org/10.1136/bmjopen-2020043954).

Received 18 August 2020

Revised 21 December 2020

Accepted 21 April 2021

\section{SLinked}

- http://dx.doi.org/10.1136/ bmjopen-2020-047185

Check for updates

(c) Author(s) (or their employer(s)) 2021. Re-use permitted under CC BY-NC. No commercial re-use. See rights and permissions. Published by BMJ.

${ }^{1}$ Manson Unit, Médecins Sans Frontières, London, UK ${ }^{2}$ Department of Sociology, University of Sussex, Brighton, UK

${ }^{3}$ Clinical Research Department London School of Hygiene \& Tropical Medicine, London, UK

Correspondence to Mrs Beverley Stringer; beverley.stringer@Iondon.msf. org

\section{ABSTRACT}

Introduction People living with multidrug-resistant tuberculosis currently have few options for effective treatment and cure. Regimens that are available are toxic, may involve injections and take up to 2 years to complete treatment, with success rates as low as $50 \%$. The TBPRACTECAL trial is evaluating shorter, more tolerable regimens of oral drugs; we detail the substudy within this trial, PRACTECAL-PR0, which aims to evaluate patient experiences and perspectives on treatment, to understand outcomes more fully.

Methods and analysis We are conducting a mixedmethods evaluation within both investigational and standard of care arms within the TB-PRACTECAL trial, using sequential quality of life $(\mathrm{Q} o \mathrm{~L})$ surveys and indepth interviews. Data collection involves the Short Form 12 (SF-12) and St George's Respiratory Questionnaire (SGRQ), collected at up to four fixed timepoints, from baseline, to up to 12 months later. Healthy volunteers will be surveyed to establish locally relevant controls. We will also purposively sample participants for qualitative data collection and analysis, to provide rich explanation of QoL scores. The study will be implemented in all six TB-PRACTECAL study sites in Uzbekistan, South Africa and Belarus. QoL surveys will be scored and analysed according to SF-12 and SGRQ developers' manuals. Differences between scores at baseline and later timepoints will be evaluated as well as graphical exploration of group score trajectories of investigational and standard of care arms.

Ethics and dissemination Ethics approval was obtained from the Médecins Sans Frontières Ethics Review Board. Local ethics approval has been obtained in Uzbekistan, Belarus and South Africa. Results of the substudy will be shared with local health authorities, the WHO and submitted for publication in a peer-reviewed journal. Trial registration number NCT03942354; Pre-results.

\section{INTRODUCTION}

Tuberculosis (TB) global epidemiology

TB remains the deadliest infectious disease globally, with mortality estimates exceeding

\section{Strengths and limitations of this study}

- This study aims to be one of the first randomised tuberculosis trials to incorporate patient perspectives on their experience of investigational treatments and compare quality of life scores with standard of care participants and healthy controls.

- Analysis includes in-depth interviews alongside standardised quality of life surveys.

- The study will detail how a novel regimen is experienced in diverse populations and contexts, covering some of the most challenging scenarios for multidrug-resistant tuberculosis treatment.

- Our findings will enable description of the utility of Short Form 12 and St George's Respiratory Questionnaire survey tools in populations living with tuberculosis.

- Limitations of the mixed-methods substudy include the relatively small sample size.

those for both HIV and malaria. The emergence of multidrug-resistant tuberculosis (MDR-TB), defined as disease caused by $\mathrm{Myco-}$ bacterium tuberculosis resistant to at least isoniazid and rifampicin, has complicated global efforts to control the epidemic. Approximately 500000 cases of MDR-TB occur globally each year, representing nearly $5 \%$ of the world's annual TB burden. ${ }^{1}$ Currently, around $20 \%$ of patients diagnosed with MDR-TB are on treatment, and there is an urgent need to scale up treatment programmes. ${ }^{2}$ Scale-up is being severely hampered by financial, political, logistical and technical obstacles, with one of the most important challenges being the nature of current standard of care regimens. ${ }^{3}$ Current regimens used to treat MDR-TB have poor efficacy; the most recent meta-analysis of treatment outcomes for pulmonary MDR-TB suggested that only 
$61 \%$ of patients had successful outcomes, $8 \%$ had failure or relapse and $14 \%$ died. ${ }^{4}$ Low treatment effectiveness, combined with high costs and implementation difficulties are preventing many national TB programmes from offering treatment for MDR-TB. ${ }^{5}$ This in turn fuels the spread of further MDR-TB infections. ${ }^{6}$ There is a global need for an improved treatment regimen for MDR-TB that is efficacious, safe, tolerable, and that can be implemented in a variety of epidemiological settings. Given the high rates of HIV co-infection among certain populations of patients with MDR-TB, ${ }^{7}$ it is imperative that patients with HIV be included in any evaluations of new treatment regimens.

\section{Patient-reported outcome (PRO) measures}

PROs are useful in evaluating the effectiveness of many medical interventions from the patient's perspective, which then can help fulfil critical considerations, such as shared decision-making, and ensuring greater user satisfaction with services. ${ }^{8}$ By using PROs in the PRACTECAL study, we will be able to assess participant progress and clinical outcomes with regards to quality of life (QoL). Most PRO measures (PROMs) used in clinical trials currently use quantitative measures only. Questionnairestyle PROMs, or QoL measures, can be generically focused, (eg, the Short Form (SF) 36, SF-12 and SF-6) ${ }^{9}$ or condition/disease-specific (eg, the St George's Respiratory Questionnaire, SGRQ), ${ }^{10}$ a respiratory QoL instrument formulated for use in chronic obstructive pulmonary disease. $^{11}$ Routine use of PROs to inform healthcare policy for a range of long-term conditions shows that use of both qualitative and quantitative methods of capturing PROs is preferable. ${ }^{12}$ We anticipate that adding participant in-depth interviews to QoL surveys will enrich our understanding from a patient perspective on the acceptability of this novel treatment and will offer detail at a country-specific level.

\section{QoL measures in tuberculosis}

Currently, work on developing QoL measures specific to $\mathrm{TB}$ is in its infancy. However, some meaningful data have been collected, and this has informed proposals that at least one PROM used must capture all health-related physical impairment (eg, not be organ-specific or systemspecific); that PROMs should be able to evaluate psychological morbidity, an issue especially pertinent for patients with MDR-TB; and that PROMs should be culturally and linguistically appropriate for the study population and to include evaluation of social role limitations and stigma. ${ }^{13}$

A systematic review of the impact of TB and the effects of treatment on patients' QoL showed that the SF-36 was the most commonly used measure to capture PROs. ${ }^{14}$ In China, SF-36 scores of patients diagnosed with TB indicated poor QoL before treatment, but these significantly improved during treatment. ${ }^{15}$ Being mindful of participant burden, we chose to use the SF-12 which accurately reproduces the two summary component scores (ie, physical and mental health) of the SF-36. ${ }^{16}$ Additionally, the qualitative interviews will explore further these domains.

The SGRQ appears to be an effective tool to assess morbidity-related QoL during treatment for people who live with TB, alongside measures of lung function, clinical improvements, chest X-ray findings and adverse events. ${ }^{17}$ QoL measures have also been used specifically to test impairment after microbiological cure, showing the importance of such measures in assessing health outcomes that are not apparent through biological measurement. ${ }^{18}$ Both physical and QoL measures demonstrate that TB appears to lead to residual disability among ambulatory patients in whom treatment outcomes may have been considered successful. ${ }^{13} 18$

\section{Qualitative PRO measures in tuberculosis}

Using qualitative methods to assess QoL for people who live with TB will include examining areas such as general health perceptions, somatic sensation or pain, psychological health, spiritual well-being, and physical, social and role functioning. ${ }^{13}$ Other published qualitative work in relation to patient perspectives has examined perceptions of self-administered TB treatment and adherence. ${ }^{19} 20$ However, there is less known about trial participant experiences and QoL using qualitative data for a new TB regimen. We hope this adds to emerging work, for example, on children and their care givers acceptance of a fixed dose regimen for TB in South Africa. ${ }^{21}$

\section{Design of the TB-PRACTECAL trial}

TB-PRACTECAL is a multicentre, open-label, phase IIIII randomised trial evaluating exclusively oral 6-month long regimens containing bedaquiline, pretomanid, linezolid only, with moxifloxacin or with clofazimine, for the treatment of microbiologically confirmed pulmonary MDR-TB and extensively drug-resistant-TB.

PRACTECAL-PRO is a substudy of the TB-PRACTECAL trial and aims to answer questions relating to adult patients' QoL while taking novel TB treatment in Uzbekistan, South Africa and Belarus.

\section{Objectives of PRACTECAL-PRO}

The TB-PRACTECAL trial assumes that even if the investigational arms show non-inferior efficacy and safety, as compared with standard of care, patients will likely prefer shorter, exclusively oral regimens with a lower pill count. We hypothesise that QoL scores in both investigational arm and standard of care patients will be worse than those of healthy controls at baseline. By using these measures, we will be adding to the available data on QoL in patients being treated for TB and will contribute data to their utility in TB clinical trials.

In the substudy, we will therefore explore these assumptions through the following objectives:

Primary objectives:

1. To assess quantitatively QoL measures for patients within the trial, from baseline to 12 months, including 
those treated in investigational arms as well as the standard of care arm.

2. To describe qualitatively patient satisfaction and experience with trial treatments in the investigational arms. Secondary objectives:

1. To understand what factors enable a novel treatment regimen to be tolerated or rejected by patients.

2. To evaluate utility of the SGRQ and SF-12 questionnaires, and qualitative methods within TB clinical trials.

\section{METHODS AND ANALYSIS \\ Overall study design}

PRACTECAL-PRO uses a mixed-methods approach, with QoL surveys and in-depth interviews being employed at different timepoints over the 12-month intervention period. It is expected that the qualitative interviews will allow for a more in-depth explanation of the quantitative survey data. QoL is assessed quantitatively within all trial arms, from baseline to 12 months with selected participants being interviewed at baseline, 3-6 months and at 12 months.

\section{Patient and public involvement}

Patients and, where feasible, the wider community have been engaged in setup and implementation stages of the main TB-PRACTECAL trial. ${ }^{22-24}$ Tools not available in the local languages are not only translated but also undergo cognitive debriefing by the teams and patients locally.

\section{Settings}

TB-PRACTECAL and the PRACTECAL-PRO substudy is being conducted in six sites, in three countries; Uzbekistan, South Africa and Belarus. In Uzbekistan, the trial is taking place in Tashkent City and six rayons (districts) in Karakalpakstan, Western Uzbekistan, specifically Nukus City, Nukus, Takhiatash, Chimbay, Kegeily and Xodjeli rayons. Implementation of the trial in Uzbekistan is being conducted by the Republican Specialised Scientific-Practical Medical Centre for Phthisiology and Pulmonology of the Republic of Uzbekistan. In South Africa, the trial is being conducted in Doris Goodwin and Don McKenzie Hospitals through the Tuberculosis and HIV Investigative Network (THINK), and Helen Joseph Hospital through the University of Witwatersrand's Clinical HIV Research Unit. In Belarus, the trial is taking place in Minsk City and Oblast, implemented by the Republican Scientific and Practical Centre for Pulmonology and Tuberculosis of the Republic of Belarus.

\section{Implementation timelines}

The study started recruitment in Belarus in October 2019 is currently recruiting in Karakalpakstan, Uzbekistan and THINK, South Africa. Recruitment completion is expected in mid-2021 and final follow-up and results are expected at the end of 2022.

\section{Sampling}

With the increasing use of QoL measures in research, historical datasets are now becoming more readily available to help guide sample size estimation and timing of surveys. ${ }^{25-29}$ Although there are published studies examining QoL changes for people with $\mathrm{TB}$, most do not inform power calculations. We recognise that it is likely that there will not be adequate power to formally detect any differences between the SGRQ in those receiving investigational treatments, and those receiving standard of care over time, however, we aim to carry out a graphical exploration of group trajectories, plotting means and 95\% CIs by group at each timepoint. This will enable us to explore whether there is any suggestion that $\mathrm{QoL}$ improves more quickly in patients who complete treatment earlier. From a review of the literature on QoL measures for tuberculosis, it appears that sample sizes are often based on a prospective cohort design, giving a projected sample size of around 100-200 patients, we are confident that the TB PRACTECAL-PRO will recruit this number.

For survey completion, we aim to recruit 54 patients in the investigational arms and 54 patients in the standard of care arm, across the three countries; 108 patients in total. All patients (interventional arm and standard therapy) will complete measures at baseline, 3 months, 6 months and 12 months. Where a recruited patient is discontinued from the trial, we will recruit an additional participant to achieve our intended sample at baseline of at least 54 investigational-arm patients and 54 standard of care patients across all sites. If numbers are likely to exceed this, we aim to keep recruiting to build a larger cohort.

Survey data from 108 healthy controls from the general population in the three study countries will be collected at one timepoint only, matched as closely as possible to the age and sex profile of trial patients. Each site will opportunistically identify participants from the community setting which may include personal contacts and colleagues not working on the PRACTECAL study. The healthy control will be screened for TB symptoms using a symptom screening tool outlined in trial standard operating procedures; only those who screen-negative will participate. For those who screen-positive, we will offer further investigation and treatment using established programmatic protocols. Additionally, we will ask each potential healthy control to tell the investigator if they consider themselves generally healthy and with no significant illnesses. Prospective participants reporting any health problems will also be excluded from the substudy.

To explore more thoroughly patient experiences across the full range of QoL scores and understand the effects of and tolerance to novel TB treatments, we will use purposive sampling to invite intervention arm participants to take part in an in-depth interview. We aim to complete up to 54 interviews across the three countries (ie, 18 per country). Selection will be based on the responses to baseline survey questionnaires; we will select patients with scores indicating a very poor QoL, those with QoL scores in the mid-range and those with scores indicating a very high QoL. 
We will seek to interview a balance of men and women, with a range of ages across all trial sites, and aim for equal numbers of patients across the different intervention arms. We aim to select an equal number of participants from each of the three trial treatment regimens at each time point while also allowing some flexibility should one of the investigational arms close early. Previous experience of similar studies has established sample size as around 12 interviews as a working figure for homogenous group selection. ${ }^{30}$ All in-depth interviews will be conducted in the local language, audio recorded and transcribed verbatim, with voluntary informed consent, in a private setting within outpatient clinics during scheduled visits. All interviews will be translated into English by local translators.

\section{Participants}

Substudy participants all have MDR-TB and will have been recruited from the main PRACTECAL study. As part of existing trial procedures, participants will be invited to take part voluntarily using an information sheet and consent form about the purpose of the study in their native language. Participants will be informed of their right to withdraw from the study at any time and the limits of confidentiality will be made explicit in the information sheet. We will conduct in-depth interviews at three timepoints during participation in the trial: (1) at or around baseline; (2) 3-6 months after therapy in the trial and (3) 12 months into the programme, that is, after treatment has been completed. Recruitment to in-depth interviews will close when data saturation occurs; that is, when no new information is being generated from subsequent interviews. ${ }^{31}$

\section{Instruments}

\section{Surveys}

The SGRQ, a disease-specific 50-item questionnaire scored in three domains, has been shown to be an effective tool for measuring the impact of airway-obstructing disease on QoL, and has been used to evaluate QoL for $\mathrm{TB}$ as well as for other respiratory diseases. ${ }^{29} \mathrm{~A}$ generic health-related QoL tool, the SF-12, is shorter than the SGRQ and was originally designed to reduce respondent burden when completing QoL surveys for people with chronic conditions, while still achieving minimum standards of precision for purposes of group comparisons involving multiple health dimensions. ${ }^{32}$ Previously translated SGRQ is available for use in Belarus and South Africa, with two translated questionnaires for Karakalpak and Uzbek required. We will also carry out quantitative data collection using the SGRQ and SF-12 questionnaires in Karakalpakstan, Uzbekistan, by translating questionnaires into Karakalpak. The SF-12 is already available in English, Russian, Sesotho and Zulu, with two translated questionnaires for Karakalpak and Uzbek.

For survey tools not available in the local language, certified translations will be obtained by working in collaboration with survey developers using an agreed cognitive debriefing protocol with a small number of patients receiving TB therapy. Local clinic workers will be trained to use questionnaires prior to the study, allowing for pretesting of tools. Data quality control and cleaning will be done in real time, and feedback and follow-up supervision will take place weekly.

\section{Interviews}

Topic guides have been developed from the results of a previously conducted literature review, ${ }^{5} 6131433$ also including questions arising from survey results. Topic areas include general health perceptions, physical health, somatic sensation or pain, side effects of drugs, benefits of treatment, hassles of therapy and so on. Topic guides will be pretested. ${ }^{34}$

In-depth interviews will be undertaken by the trial principal investigator and a locally trained researcher. Where possible, interviews will be conducted in participants' own native languages, but where this is not feasible, interviews will be done in English, with simultaneous translation. Interpreters will be trained and checked for proficiencies to support the principal investigator and locally trained researchers. All researchers will document field notes during fieldwork, detailing insights and observations that develop over time and through repeated analysis of events, activities and interactions. This aims to enhance understanding of data collected through in-depth interviews, increasing the strength of results. ${ }^{35}$

\section{Data analysis}

In analysing our data, we will:

- Compare baseline scores between trial patients (all investigational arm patients, plus standard of care patients) with healthy controls.

- Assess changes in scores over time in patients in intervention arms and patients in the standard of care arm.

- Assess the utility of SGRQ and SF-12 instruments in a TB clinical trial.

- Use qualitative data to more fully understand patient experiences of a shortened trial treatment regimen.

\section{Quantitative data}

Data will be scored using the developers' scoring manuals.

\section{Qualitative data}

Transcripts will be analysed thematically, aiming to identify and explain patterns in the data. ${ }^{36}$ Field notes made throughout the fieldwork period will be used to guide data analysis. Transcribed interview data will be broken into units of meaning (ie, a word, phrase, sentence or paragraph), and open or tentative codes will be applied to those units. Axial coding will be used to compare codes across the dataset to identify the relationships between them and to derive core codes. Selective coding will then be undertaken whereby the core codes will be repeatedly applied to transcripts leading to identification and development of latent patterns and themes. Negative cases (ie, data that challenges the emerging analysis) will 
be examined in order to test emerging themes and to explain why these cases are different. ${ }^{35}$

A coding dictionary and analytic memos will be developed and scrutinised by a minimum of two team members to enhance analytic credibility. Selected anonymised interview excerpts or case studies will be drawn out to ensure the individual 'stories' are not lost and to explore how the themes inter-relate between and within cases. ${ }^{37}$

The results of our substudy will give insights about the benefits and risks of treatment through greater understanding of participant opinions and experience, which might otherwise be overlooked. The methods used here will help assess patient perspectives, potentially demonstrating how patient priorities can be evaluated in complex trial intervention. Adding patient perspectives is beneficial to supporting licensing claims for new medicines and to influence the development of health policy, including decisions about the cost-effectiveness of treatment. ${ }^{38}$

\section{Ethics and dissemination}

The study will be conducted according to the ethical principles as defined in the Declaration of Helsinki. The protocol and corresponding documents were reviewed and approved by the Médecins Sans Frontières (MSF) Ethics Review Board, reference number 1541b. Local ethical approval has been obtained from relevant agencies in each study setting. In South Africa, this includes PharmaEthics, University of Witwatersrand Human Research Ethics Committee; in Uzbekistan, the Ethical Committee of the Ministry of Health of the Republic of Uzbekistan; in Belarus, the Ethics Committee of the State Institution Republican Scientific and Practical Centre of Pulmonology and Tuberculosis, and the Centre of Expertise for Testing in Healthcare.

Request for consent for participants to join the substudy will follow their agreement to join the wider TB-PRACTECAL trial. Information given, and informed consent processes will be similar across study sites. Participation in PRACTECAL-PRO is optional for patients who have already consented to the main TB-PRACTECAL trial, and consent for the substudy is obtained in addition to that for the main trial.

Printed and electronic versions of the final report will be provided to all partners involved in this project. A meeting will be held with participants to discuss the emergent findings and to gain their feedback and thoughts on these. A study manuscript will be produced and submitted for publication in a peer-reviewed scientific journal, and authorship of any publication will be based on the Uniform Requirements for Manuscripts Submitted to Biomedical Journals as defined by the International Committee of Medical Journal Editors.

Discussions will be held with national ministries of health, MSF trial team contacts and coordination teams regarding the influence of study findings on future programme activities. Research methodology and results will also be presented at scientific conferences.
Twitter Bern-Thomas Nyang'wa @docwak77

Acknowledgements Emma Veitch, freelance medical editor for MSF, UK, provided editorial assistance and her work was funded by MSF UK. Professor Kevin Schwartzman, director, Respiratory Division, McGill University and McGill University Health Centre peer reviewed the study protocol. Dr Heidi Lempp, Reader in Medical Sociology, Faculty of Life Sciences \& Medicine, Kings College London, peer reviewed the study protocol. Professor Elizabeth Allen, Head of Medical Statistic Department, London School of Hygiene and Tropical Medicine contributed to the statistical analysis component of the study protocol. Paul W. Jones, Frances H. Quirk, Chloë M. Baveystock, Department of Medicine, St. George's Hospital Medical School, London, UK, developed the SGRQ. We would like to thank principal investigators at each site for overseeing implementation: Dr Varvara Solodovnikova, Republican Research and Practical Centre for Pulmonology and Tuberculosis, Belarus. Professor Parpieva Nargiza, country principal investigator, Republican Specialised Scientific-Practical Medical Centre of Tuberculosis And Pulmonology, Tashkent, Uzbekistan. Dr Liverko Irinve, Republican Specialised Scientific-Practical Medical Centre for Phthisiology and Pulmonology of the Republic of Uzbekistan, Tashkent, Uzbekistan. Dr Tigay Zinaida, Republican TB Hospital 2, Nukus, Uzbekistan. Dr Mohammed Rassool, Wits Health Consortium CHRU, Helen Joseph Hospital, South Africa. Dr Ronelle Moodliar, THINK: Tuberculosis \& HIV Investigative Network, Doris Goodwin and Don McKenzie Hospitals, South Africa. We would also like to thank those involved in the cognitive debriefing work towards the SGRQ translations.

Contributors BS is the principal investigator of the study, has contributed to concept and protocol development and is responsible for its implementation. $\mathrm{KL}$ is co-investigator, and has contributed to conceptualisation and protocol development with responsibility for supervision of quantitative data collection and analysis of the PRO substudy. B-TN is chief investigator and the sponsor's representative for the TB-PRACTECAL trial, and defined study scope, contributed to protocol development and sign-off. $\mathrm{NJ}$ is a research associate for the TB-PRACTECAL trial and contributed to review and finalisation of the manuscript.

Funding This work was supported by Médecins sans Frontières.

Competing interests None declared.

Patient and public involvement Patients and/or the public were involved in the design, or conduct, or reporting, or dissemination plans of this research. Refer to the Methods section for further details.

Patient consent for publication Not required.

Provenance and peer review Not commissioned; externally peer reviewed.

Open access This is an open access article distributed in accordance with the Creative Commons Attribution Non Commercial (CC BY-NC 4.0) license, which permits others to distribute, remix, adapt, build upon this work non-commercially, and license their derivative works on different terms, provided the original work is properly cited, appropriate credit is given, any changes made indicated, and the use is non-commercial. See: http://creativecommons.org/licenses/by-nc/4.0/.

ORCID iDs

Beverley Stringer http://orcid.org/0000-0003-1859-6650

Nicola James http://orcid.org/0000-0002-1269-9811

Bern-Thomas Nyang'wa http://orcid.org/0000-0001-9915-5878

\section{REFERENCES}

1 World Health Organization. Global tuberculosis report 2019, 2019.

2 WHO. Drug resistant- TB, surveillance and response. J Chem Inf Model 2013;53:1689-99.

3 Hughes J, Brigden G, Nyang'wa B. Principles for designing future regimens for multidrug-resistant tuberculosis principles for designing future regimens for multidrug- resistant tuberculosis. Bull WHO 2014;92:68-74.

4 Ahmad N, Ahuja SD, Akkerman OW. Collaborative group for the meta-analysis of individual patient data in MDR-TB treatment-2017. Lancet 2018;392:821-34.

5 Bauer M, Leavens A, Schwartzman K. A systematic review and metaanalysis of the impact of tuberculosis on health-related quality of life. Qual Life Res 2013;22:2213-35.

6 Fitzpatrick C, Floyd K. A systematic review of the cost and cost effectiveness of treatment for multidrug-resistant tuberculosis. Pharmacoeconomics 2012;30:63-80.

7 Pontali E, Matteelli A, Migliori GB. Drug-resistant tuberculosis. Curr Opin Pulm Med 2013;19:266-72. 
8 Wolpert M, Jacob J, Napoleone E. Child-and parent-reported outcomes and experience from child and young people's mental health services 2011-2015, 2016.

9 RAND. 36, 12 and 6 -item short form survey scoring instructions, 2012.

10 Jones PW. Patients original English version. St. Georges Questionnaire 2010;44:1-10.

11 Daudey L, Peters JB, Molema J, et al. Health status in COPD cannot be measured by the St George's Respiratory Questionnaire alone: an evaluation of the underlying concepts of this questionnaire. Respir Res 2010;11:1-7.

12 Neale J, Strang J. Blending qualitative and quantitative research methods to optimize patient reported outcome measures (PROMs). Addiction 2015;110:1215-6.

13 Brown J, Capocci S, Smith C, et al. Health status and quality of life in tuberculosis. Int J Infect Dis 2015;32:68-75.

14 Guo N, Marra F, Marra CA. Measuring health-related quality of life in tuberculosis: a systematic review. Health Qual Life Outcomes 2009;7:14-10.

15 Chamla D. The assessment of patients' health-related quality of life during tuberculosis treatment in Wuhan, China. Int $J$ Tuberc Lung Dis 2004;8:1100-6.

16 Jenkinson $C$, Layte $R$, Jenkinson $D$, et al. A shorter form health survey: can the SF-12 replicate results from the SF-36 in longitudinal studies? J Public Health Med 1997;19:179-86.

17 Hansel NN, Wu AW, Chang B, et al. Quality of life in tuberculosis: patient and provider perspectives. Qual Life Res 2004;13:639-52.

18 Maguire GP, Anstey NM, Ardian M, et al. Pulmonary tuberculosis, impaired lung function, disability and quality of life in a high-burden setting. Int J Tuberc Lung Dis 2009;13:1500-6.

19 Stringer B, Lowton K, Tillashaikhov M, et al. They prefer hidden treatment: anti-tuberculosis drug-taking practices and drug regulation in Karakalpakstan. Int J Tuberc Lung Dis 2016;20:1084-90.

20 Horter S, Stringer B, Greig J, et al. Where there is hope: a qualitative study examining patients' adherence to multi-drug resistant tuberculosis treatment in Karakalpakstan, Uzbekistan. BMC Infect Dis 2016;16:362.

21 Wademan DT, Busakwe L, Nicholson TJ, et al. Acceptability of a firstline anti-tuberculosis formulation for children: qualitative data from the shine trial. Int J Tuberc Lung Dis 2019;23:1263-8.

22 The BMJ. Engaging communities in tuberculosis research: the experience of the TB-PRACTECAL trial. Available: https://blogs.bmj. com/bmj/2018/11/09/engaging-communities-tuberculosisresearchexperience-practecal-trial/ [Accessed 16 Dec 2019].

23 Babaniyazov A, Nyang'wa BT, Pardington N. 'Trial and Error' operational reflections on the set-up of a community engagement programme for a phase ii/iii clinical trial to identify new MDR-TB treatments in Uzbekistan. Médecins Sans Frontières Scientific Day South Asia 2016.

24 Wharton-Smith A, Gray N, Stringer B. Optimising recruitment to a TB clinical trial in Uzbekistan [version 1; not peer reviewed]. F1000Research 2020;9:466.

25 Gandek B, Ware JE, Aaronson NK, et al. Cross-validation of item selection and scoring for the SF-12 health survey in nine countries: results from the IQOLA project. International quality of life assessment. J Clin Epidemiol 1998;51:1171-8.

26 Patel ARet al. The validity of the SF-12 and SF-6D instruments in people living with HIV/AIDS in Kenya. Health Qual Life Outcomes 2017;15:1-9.

27 Hayes CJ, Bhandari NR, Kathe N, et al. Reliability and validity of the medical outcomes study short form-12 version 2 (SF-12v2) in adults with non-cancer pain. Healthcare 2017;5:22

28 Roberts B, Browne J, Ocaka K, et al. The reliability and validity of the SF-8 with a conflict-affected population in northern Uganda. Health Qual Life Outcomes 2008;6:108-10

29 Pasipanodya JG, Miller TL, Vecino M, et al. Using the St. George respiratory questionnaire to ascertain health quality in persons with treated pulmonary tuberculosis. Chest 2007;132:1591-8.

30 Guest G, Bunce A, Johnson L. How many interviews are enough? Field Method 2006;18:59-82.

31 Green J, Thorogood N. Principles and approaches in qualitative research. 3rd edn. Sage, 2014.

32 Ware JE, Kosinski M, Keller SD. SF-12 : how to score the SF-12 physical and mental health summary scales. New Eng Med Center 2002.

33 Holmberg C, Karner JJ, Rappenecker J, et al. Clinical trial participants' experiences of completing questionnaires: a qualitative study. BMJ Open 2014;4:e004363.

34 Hurst S, Arulogun OS, Owolabi AO, et al. Pretesting qualitative data collection procedures to facilitate methodological adherence and team building in Nigeria. Int J Qual Methods 2015;14:53-64.

35 Charmaz K. Constructing Grounded theory: a practical guide through qualitative analysis. 1st edn. Sage, 2006.

36 Bradley EH, Curry LA, Devers KJ. Qualitative data analysis for health services research: developing taxonomy, themes, and theory. Health Serv Res 2007;42:1758-72.

37 Braun V, Clarke V. Using thematic analysis in psychology. Qual Res Psychol 2006;3:77-101. doi:10.1191/1478088706qp063oa

38 Fallowfield L. Human horizons series. the quality of life: the missing measurement in health care. Souvenir Press, 1990. 